\title{
Sprachstandserhebungstest für Kinder im Alter zwischen 3 und 5 Jahren (SET 3-5): Zusatzauswertung Aussprache
}

\author{
Franz Petermann, Jessica Melzer
}

\author{
Ergänzung zur Testbesprechung \\ Achhammer, B. (2018): Sprachstandserhebungstest für Kinder im Alter zwischen \\ 3 und 5 Jahren (SET 3 - 5), Frühförderung interdisziplinär, 37 (2), 97- 101
}

Die sehr differenzierte Testbesprechung auf S. 97 - 101 in Ausgabe 2/2018 der Frühförderung interdisziplinär, für die wir uns bedanken, möchten wir durch den folgenden Hinweis zur Zusatzauswertung Aussprache beim SET 3-5 ergänzen.

Mit dem SET 3 - 5 hat der Anwender die Möglichkeit auf Basis des expressiven Wortschatztests Bildbenennung auch eine Analyse der Aussprache eines Kindes durchzuführen. Bei der Entwicklung der Items wurde darauf geachtet, verschiedene Phoneme sowohl in initialer, medialer und finaler Wortstellung als auch in Konsonantenverbindung zu berücksichtigen. Durch ein solches Aussprachescreening kann eine erste Einschätzung auf phonetisch-phonologischer Ebene erfolgen. Für die Auswertung auf phonetisch-phonologischer Ebene ist es besonders wichtig, dass alle Worte vom Kind benannt werden. Aus diesem Grund wird ein Kind gebeten, Wörter nachzusprechen, die noch nicht im Wortschatz des Kindes vorhanden sind und somit nicht benannt werden konnten.

Wenn die Äußerungen eines Kindes protokolliert werden, kann durch die Einteilung in Substitution, Elision, Addition und Phonetische Abweichung bereits auf dem Protokollbogen festgehalten werden, welche Laute vorhanden sind und welche im Phonembestand des Kindes fehlen. Auf dieser Basis kann für das Kind im Anschluss ein Phoneminventar erstellt werden. Hierbei werden die einzelnen Phoneme der Zielwörter betrachtet. Angepasst an die Reihenfolge im Protokollbogen werden im Phoneminventar die Zielwörter zusammen mit der entsprechenden Lautschrift nach dem Internationalen Phonetischen Alphabet (IPA) aufgeführt.

Im Anschluss wird, unter Verwendung der Auswertungstabelle des Phoneminventars auf der letzten Seite, die Summe der einzelnen Phoneme in ihren entsprechenden Positionen bzw. in ihrer Konsonatenverbindung bestimmt. Nachdem dies für alle Phoneme berechnet wurde, werden die Summenwerte für die verschiedenen Positionen der Laute im Wort als auch die Summen der einzelnen Phoneme unabhängig von ihrer Position bestimmt. Darüber hinaus sollte eine Analyse der phonetisch-phonologischen Prozesse durchgeführt werden. Auf dem Befundbogen sind alle physiologisch-phonologischen Prozesse, die im Spracherwerb monolingual deutschsprachiger Kinder im Alter zwischen 2;0 und 4;11 Jahren auftreten, aufgeführt. 
Damit sich eventuelle Auffälligkeiten im phonologischen bzw. phonetischen Bereich nicht auf die Bewertung in anderen Bereichen auswirken und somit das Testergebnis verschlechtern, z. B. in der auditiven Merkfähigkeit (erhoben durch das Nachsprechen von Kunstwörtern), wird der Untertest Bildbenennung zu Beginn der Testdurchführung mit dem Kind bearbeitet. Auffälligkeiten, die sich hier zeigen, können dann für die Auswertung in den anderen Bereichen kontrolliert werden. So findet sich z. B. für den Untertest 11 Kunstwörter nachsprechen in der Durchführungsanleitung ein entsprechender Hinweis.

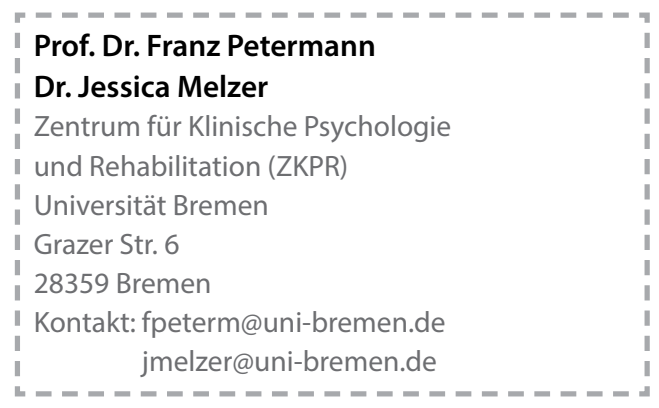

\section{Literatur}

Petermann, F., Rißling, J. K., Melzer, J. (2016): Sprachstandserhebungstest für Kinder im Alter zwischen 3 und 5 Jahren (SET 3-5). Hogrefe, Göttingen 